UPR-1037-T, DCPT-03/19

hep-th/0304150

\title{
Holography beyond the horizon and cosmic censorship
}

\author{
Thomas S. Levi ${ }^{1 *}$ and Simon F. Ross ${ }^{2 \dagger}$ \\ ${ }^{1}$ David Rittenhouse Laboratories, University of Pennsylvania, \\ Philadelphia, PA 19104, USA \\ ${ }^{2}$ Centre for Particle Theory, Department of Mathematical Sciences \\ University of Durham, South Road, Durham DH1 3LE, U.K.
}

\begin{abstract}
We investigate the description of the region behind the event horizon in rotating black holes in the AdS/CFT correspondence, using the rotating BTZ black hole as a concrete example. We extend a technique introduced by Kraus, Ooguri and Shenker [1, based on analytically continuing amplitudes defined in a Euclidean space, to include rotation. In the rotating case, boundary amplitudes again have two different bulk descriptions, involving either integration only over the regions outside the black holes' event horizon, or integration over this region and the region between the event horizon and the Cauchy horizon (inner horizon). We argue that generally, the holographic map will relate the field theory to the region bounded by the Cauchy horizons in spacetime. We also argue that these results suggest that the holographic description of black holes will satisfy strong cosmic censorship.
\end{abstract}

\section{Introduction}

Black holes play a central role in the AdS/CFT correspondence. The original proposal of a large $N$ duality [2] was largely motivated by the successes of string theory in explaining the black hole entropy, and soon afterwards, it was realised [3] that the black hole geometries are directly related to thermal states in the gauge theory. This led to non-trivial predictions for the phase structure of the strongly-coupled gauge theory, and placed our understanding of string theory's successes in accounting for the black hole entropy on a much firmer footing. However, our understanding of the description of the black hole geometry (as opposed to its thermodynamic properties) from the dual field theory point of view remains very weak. It has proved difficult

*tslevi@student.physics.upenn.edu

†S.F.Ross@durham.ac.uk 
to address even the simplest questions about observations in a black hole spacetime from the field theory point of view. For example, we do not know how the 'one-way' nature of the event horizon is (approximately) enforced in the field theory, nor do we know how to describe the observations of an observer falling across the black hole horizon.

These difficulties reflect broader problems in understanding the Lorentzian aspects of the AdS/CFT correspondence. The connection between thermodynamics of black holes and thermodynamics of gauge theory can be understood using the Euclidean black hole solutions. But in this Euclidean section, there is no region 'behind the event horizon': in positive definite signature there is in general no coordinate-independent notion of an event horizon. The usual analytic continuation back to Lorentzian signature maps the Euclidean section onto the region outside the event horizon of an eternal black hole. It has sometimes been suggested that this implies that the dual field theory describes only physics in the region outside the event horizon. However, as argued in [4, this seems very unlikely: there is a Cauchy surface for the black hole spacetime which lies entirely outside the event horizon. Thus, if the dual field theory provides a complete description of the physics in this region, it will also determine what is happening behind the horizon. That is, degrees of freedom which pass behind the event horizon should still be encoded in the dual field theory in some way, which could involve some form of black hole complementarity [5, 6].

Attempts to explore this encoding were made in [7, 8, 9, 10]. In [7, 8], attention was focused on the description of black holes formed from collapse and on the geon, so the geometry has a single asymptotic boundary, and the full geometry including the region behind the horizon should be described by some appropriate state in the dual field theory. One obstacle to progress in these cases was that the correct state in the field theory is not easily identified. In [9], the eternal BTZ black hole was considered, and a dual description of the geometry in terms of a specific entangled state of the two CFTs living on the two asymptotic boundaries of the spacetime was proposed (building on previous work [11, 12, 13, 14]). This provides a concrete context in which one can ask what information about the region behind the event horizon is contained in correlation functions of CFT operators in this entangled state. It was argued in [9] that correlation functions involving operators in both CFTs would indeed probe the region behind the horizon.

In [1], substantial progress in implementing these ideas was achieved by introducing two key ingredients: first, the calculation of higher $n$-point correlators using bulk-boundary propagators was considered. This involves an interaction vertex which is integrated over the bulk, allowing the explicit identification of contributions from different regions of the spacetime. Second, a novel analytic continuation was found, which relates the integration of the bulk interaction vertex over the Euclidean black hole to an integration over the whole black hole spacetime, including the regions inside the black hole event horizon. This amplitude could also be analytically continued in the standard way, to obtain a representation in terms of an integral just over the region outside the black hole event horizon. (These alternative analytic continuations were also discussed in [15].) This technique thus provides an explicit realisation of the ideas of black hole complementarity [5, 6]. It shows that there is a representation 
in which the field theory treats the black hole singularity, and not the event horizon, as the end of the classical spacetime description.

In this paper, we will extend the analysis of [1] to consider the rotating BTZ black hole. This involves a generalisation of the analytic continuation described in [1. We will see that the field theory calculation can only be related to an integral over the region outside the inner horizon of the black hole; this suggests the region inside the inner horizon, where the singularities are visible, is unphysical from the field theory point of view.

We proceed as in [1, investigating the definition of correlation functions in the entangled CFTs on the two asymptotic boundaries of the rotating black hole by analytic continuation from a Euclidean solution. In the bulk description, a boundary correlation function is calculated by evaluating appropriate Feynman diagrams constructed from bulk-boundary and bulk-bulk propagators, with the interaction vertices integrated over the bulk spacetime. As we analytically continue the boundary points from the Euclidean to the Lorentzian section, we must deform the contour of integration for these interaction vertices in some way.

We will investigate two different ways of deforming this contour, which lead to different representations of the same Lorentzian correlation function. In the first case, we deform in the usual way, taking the integration over the Euclidean spacetime to be an integration over the regions outside the black hole event horizons. We then find a new 'Kruskal-like' coordinate system, which allows us to define a deformation of the contour as we analytically continue which can be interpreted as giving an integration of the interaction vertices over the whole region between the past and future Cauchy horizons (inner horizons), including both the regions between the inner and outer horizons and the exterior asymptotic regions. Thus, in this case, the boundary correlation function can be related to an integral over the Cauchy development of a Cauchy surface connected to the asymptotic boundaries. There appears to be no representation in which the computation of boundary observables involves the spacetime region beyond the Cauchy horizon. The field theory seems to see the Cauchy horizon (and not the event horizon) as a natural boundary. This supports the argument given previously, that the region inside the event horizons must be described by the field theory because it is included in this Cauchy development.

There is another reason for expecting the spacetime to be cut off at the Cauchy horizon: an observer who crosses it will see the naked timelike singularity inside the black hole, leading to a violation of strong cosmic censorship [16]. The fact that the CFT only appears to describe the region outside the Cauchy horizon may therefore be interpreted as an interesting manifestation of cosmic censorship in the AdS/CFT correspondence. It has previously been argued that violations of strong cosmic censorship in such charged black hole spacetimes are prevented by an instability of the Cauchy horizon: it is a surface of infinite blueshift [17], and is therefore unstable to generic perturbations of the exterior geometry [18. It is not clear if the fact that the CFT sees the Cauchy horizon as the end of the classical spacetime in rotating black holes is a reflection of this instability, or represents a truly independent mechanism for enforcing cosmic censorship. An important direction for future work is to look for signs of singular behaviour in the CFT associated with the region of spacetime 
near Cauchy horizons, and to understand the conjectured breakdown of the classical spacetime picture there.

In section 2, we review the rotating BTZ black hole solution, and discuss the analytic continuation relating the Euclidean and Lorentzian solutions in the standard BTZ coordinate system [19]. This analytic continuation was also recently discussed in [20], where the propagation of strings in this geometry was considered. In section 3, we introduce a new coordinate system, in which the analytic continuation relates the Euclidean section to the regions outside the event horizon and between the event horizon and the Cauchy horizon. Our discussion follows that in [1] very closely. Section 4 contains some concluding remarks and thoughts on future directions.

\section{Rotating BTZ black holes}

We will begin by reviewing the relevant features of the BTZ solution, the propagators for scalar fields on this spacetime, and the analytic continuation to define a related spacetime with positive definite metric. In static coordinates, the rotating BTZ solution of [19] is (we are setting the AdS length scale associated with the cosmological constant to one)

$$
d s^{2}=-\frac{\left(r^{2}-r_{+}^{2}\right)\left(r^{2}-r_{-}^{2}\right)}{r^{2}} d t^{2}+\frac{r^{2} d r^{2}}{\left(r^{2}-r_{+}^{2}\right)\left(r^{2}-r_{-}^{2}\right)}+r^{2}\left(-\frac{r_{+} r_{-}}{r^{2}} d t+d \bar{\phi}\right)^{2} .
$$

The parameters $r_{ \pm}$are related to the mass and angular momentum of the solution through

$$
r_{ \pm}^{2}=\frac{M}{2}\left[1 \pm\left(1-\frac{J^{2}}{M^{2}}\right)^{1 / 2}\right]
$$

so $M=r_{+}^{2}+r_{-}^{2}, J=2 r_{+} r_{-}$. This solution is locally $\mathrm{AdS}_{3}$; this is most easily seen by relating the coordinate system used in (11) to the embedding coordinates $\left(T_{1}, T_{2}, X_{1}, X_{2}\right)$ for the $\mathrm{AdS}_{3}$ hyperboloid, which satisfy $T_{1}^{2}+T_{2}^{2}-X_{1}^{2}-X_{2}^{2}=1$. They are related by

$$
\begin{gathered}
T_{1}=\sqrt{\alpha} \cosh \left(r_{+} \bar{\phi}-r_{-} t\right), \\
T_{2}=\sqrt{\alpha-1} \sinh \left(r_{+} t-r_{-} \bar{\phi}\right), \\
X_{1}=\sqrt{\alpha} \sinh \left(r_{+} \bar{\phi}-r_{-} t\right), \\
X_{2}=\sqrt{\alpha-1} \cosh \left(r_{+} t-r_{-} \bar{\phi}\right),
\end{gathered}
$$

where $\alpha=\frac{r^{2}-r_{-}^{2}}{r_{+}^{2}-r_{-}^{2}}$. In discussing physics near the horizon, it is often useful to introduce a different angular coordinate, $\phi=\bar{\phi}-r_{-} t / r_{+}$. In terms of this coordinate,

$$
d s^{2}=-\frac{\left(r^{2}-r_{+}^{2}\right)\left(r^{2}-r_{-}^{2}\right)}{r^{2}} d t^{2}+\frac{r^{2} d r^{2}}{\left(r^{2}-r_{+}^{2}\right)\left(r^{2}-r_{-}^{2}\right)}+r^{2}\left[\frac{r_{-}}{r_{+} r^{2}}\left(r^{2}-r_{+}^{2}\right) d t+d \phi\right]^{2}
$$

and the relation to the embedding coordinates becomes

$$
T_{1}=\sqrt{\alpha} \cosh \left(r_{+} \phi\right)
$$



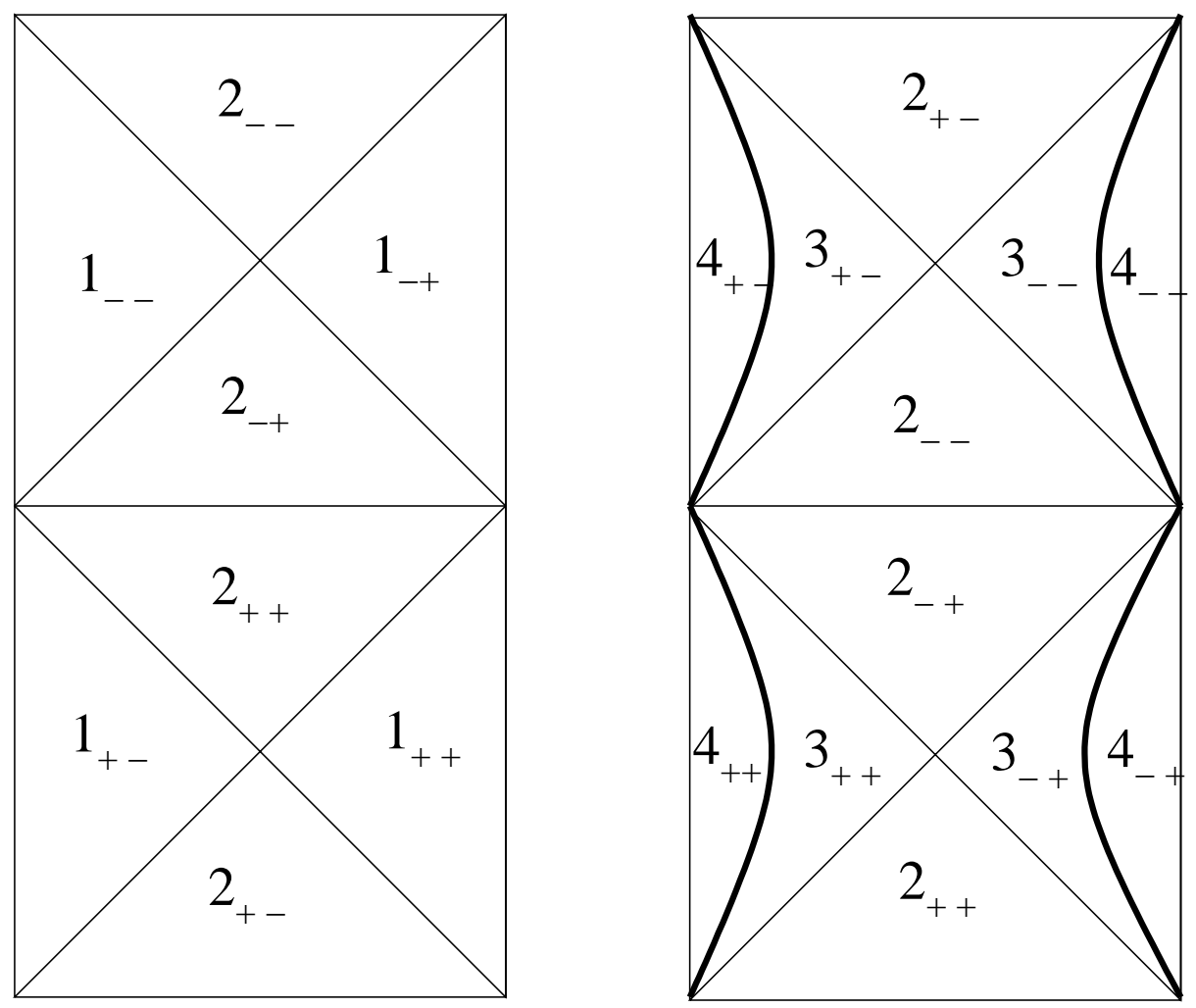

Figure 1: Slices through $\mathrm{AdS}_{3}$ in global coordinates, showing the regions of $\mathrm{AdS}_{3}$ determined by the norm of the Killing vector $\xi=\partial_{\phi}$ in the coordinates (17). In region $1, \xi \cdot \xi \geq r_{+}^{2}$; in region $2, r_{+}^{2} \geq \xi \cdot \xi \geq r_{-}^{2}$; in region $3, r_{-}^{2} \geq \xi \cdot \xi>0$; in region 4 , $\xi \cdot \xi \leq 0$.

$$
\begin{gathered}
T_{2}=\sqrt{\alpha-1} \sinh \left(\frac{r_{+}^{2}-r_{-}^{2}}{r_{+}} t-r_{-} \phi\right), \\
X_{1}=\sqrt{\alpha} \sinh \left(r_{+} \phi\right), \\
X_{2}=\sqrt{\alpha-1} \cosh \left(\frac{r_{+}^{2}-r_{-}^{2}}{r_{+}} t-r_{-} \phi\right) .
\end{gathered}
$$

This change of coordinates gives a metric where the $g_{t \phi}$ cross term vanishes at the horizon, so $\phi$ is a co-rotating coordinate near the horizon (the original coordinate system is adapted to discussing infinity; the $g_{t \bar{\phi}}$ cross term vanishes at infinity).

This spacetime is naturally divided into several regions, determined by the norm of the Killing vector $\xi=\partial_{\phi}$ along which we identify. If $\xi \cdot \xi \geq r_{+}^{2}$, we are outside the black hole's event horizon; this is region 1. If $r_{+}^{2} \geq \xi \cdot \xi \geq r_{-}^{2}$, we are between the event horizon and the Cauchy horizon; this is region 2 . If $r_{-}^{2} \geq \xi \cdot \xi>0$, we are between the Cauchy horizon and the 'singularity' of the black hole; this is region 3. Finally, in the global $\mathrm{AdS}_{3}$ space before making identifications, there is a region where $\xi \cdot \xi \leq 0$, which we call region 4 -this region is omitted from the black hole spacetime. Because we cut out region 4 , treating the surface $\xi \cdot \xi=0$ as a singularity 
of the rotating black hole spacetime, this black hole solution has no closed timelike curves. The division of global $\mathrm{AdS}_{3}$ into these regions is depicted in figure 1 .

As in [1, it will be convenient later to note that the division into regions can also be described in terms of the embedding coordinates. Since $T_{1}^{2}-X_{1}^{2}=\alpha$, and $T_{2}^{2}-X_{2}^{2}=1-\alpha$, the regions can also be distinguished by

$$
\begin{array}{rll}
\text { region } 1: & T_{1}^{2}-X_{1}^{2} \geq 0, \quad T_{2}^{2}-X_{2}^{2} \leq 0 \\
\text { region 2: } & T_{1}^{2}-X_{1}^{2} \geq 0, \quad T_{2}^{2}-X_{2}^{2} \geq 0 \\
\text { regions } 3 \& 4: & T_{1}^{2}-X_{1}^{2} \leq 0, \quad T_{2}^{2}-X_{2}^{2} \geq 0
\end{array}
$$

We also note that each of the regions consists of several disconnected components; these are conveniently distinguished by the sign of certain combinations of the embedding coordinates. We adopt the same procedure as [1], denoting the different regions by $A_{\eta_{1} \eta_{2}}$, where $A=1, \ldots, 4$ and $\eta_{1}, \eta_{2}= \pm$ are the signs of the two combinations $T_{1}+X_{1}$ and $T_{2}+X_{2}$. Thus, for example, the ordinary region outside the black hole covered by the coordinates in (11), where $T_{1}+X_{1}=\sqrt{\alpha} e^{r_{+} \phi}>0$ and $T_{2}+X_{2}=\sqrt{\alpha-1} \exp \left(\frac{r_{+}^{2}-r_{-}^{2}}{r_{+}} t-r_{-} \phi\right)>0$, is denoted $1_{++}$.

We want to investigate the calculation of field theory amplitudes using the bulkboundary propagators for a scalar field on this solution, defining the propagators by analytic continuation from a Euclidean section. The rotating BTZ metric has no Euclidean continuation per se; we cannot define a real Euclidean metric by complexifying the coordinates in the above metric. However, there is a standard analytic continuation to a Euclidean space defined by analytically continuing simultaneously in the coordinates and in the parameter $r_{-} \cdot{ }^{1}$ We set $t=-i \tau, r_{-}=-i \tilde{r}_{-}$(so $\left.\tilde{J}=i J=2 r_{+} \tilde{r}_{-}\right)$. With this analytic continuation, the metric (7) becomes

$$
d s^{2}=\frac{\left(r^{2}-r_{+}^{2}\right)\left(r^{2}+\tilde{r}_{-}^{2}\right)}{r^{2}} d \tau^{2}+\frac{r^{2} d r^{2}}{\left(r^{2}-r_{+}^{2}\right)\left(r^{2}+\tilde{r}_{-}^{2}\right)}+r^{2}\left[-\frac{\tilde{r}_{-}}{r_{+} r^{2}}\left(r^{2}-r_{+}^{2}\right) d \tau+d \phi\right]^{2} .
$$

At $r=r_{+}$, there is a conical singularity in this metric, as $g_{\tau \tau} \rightarrow 0$. We must therefore identify $\tau$ periodically with period $\Delta \tau=2 \pi r_{+} /\left(r_{+}^{2}+\tilde{r}_{-}^{2}\right)$. In this coordinate system, the Euclidean metric (13) is smooth with the separate identifications $\phi \sim \phi+2 \pi n$ and $\tau \sim \tau+m \Delta \tau$, with $m, n$ integers. One normally expects a rotating black hole to involve a twisted identification $\bar{\phi} \sim \bar{\phi}+\Omega \beta, \tau \sim \tau+\beta$, where $\beta$ and $\Omega$ are then interpreted as temperature and a rotational chemical potential respectively. In the present coordinates, this twisting is hidden in the fact that the cycles that are orthogonal at large distances are given by $\bar{\phi}$ and $\tau$, not $\phi$ and $\tau$.

Let us now consider a scalar field of mass $m$ propagating in this geometry. The bulk-boundary propagator in the BTZ black hole (11) can be obtained by the method

\footnotetext{
${ }^{1}$ Of course, global $\mathrm{AdS}_{3}$ does have a Euclidean section. If we think of the BTZ black hole as a quotient of global $\mathrm{AdS}_{3}$, this analytic continuation of $r_{-}$amounts to redefining the identification we consider as we perform the analytic continuation from the Lorentzian to the Euclidean section in $\mathrm{AdS}_{3}$.
} 
of images from the propagator in $\mathrm{AdS}_{3}$. The result is [21]

$$
\begin{aligned}
K^{1_{++} 1_{++}}\left(x, b^{\prime}\right)=\sum_{n=-\infty}^{\infty} \quad\{ & -\sqrt{\alpha-1} \cosh \left[r_{+} \Delta t-r_{-}(\Delta \bar{\phi}+2 \pi n)\right] \\
& \left.+\sqrt{\alpha} \cosh \left[r_{+}(\Delta \bar{\phi}+2 \pi n)-r_{-} \Delta t\right]\right\}^{-2 h_{+}}
\end{aligned}
$$

where $x=(t, \bar{\phi}, r)$ is the bulk point, $b^{\prime}=\left(t^{\prime}, \bar{\phi}^{\prime}\right)$ is the boundary point, $\Delta t=t-t^{\prime}$, $\Delta \bar{\phi}=\bar{\phi}-\bar{\phi}^{\prime}$, and $2 h_{+}=1+\sqrt{1+m^{2}}$. We will rewrite this in the coordinate system (7), and introduce the notation $\Delta \phi_{n} \equiv \Delta \phi+2 \pi n$ for convenience, giving

$$
\left.K^{1_{++} 1_{++}}\left(x, b^{\prime}\right)=\sum_{n=-\infty}^{\infty}\left\{-\sqrt{\alpha-1} \cosh \left[\frac{\left(r_{+}^{2}-r_{-}^{2}\right)}{r_{+}} \Delta t-r_{-} \Delta \phi_{n}\right)\right]+\sqrt{\alpha} \cosh \left(r_{+} \Delta \phi_{n}\right)\right\}^{-2 h_{+}} .
$$

In writing this form for the propagator, we have assumed that both boundary and bulk points are in region $1_{++}$, outside the horizon. To obtain the propagator for the bulk point in other regions requires analytic continuation of this propagator, as explained in [1]. For example, the propagator for the boundary point in region $1_{++}$ and the bulk point in region $2_{++}$is given by

$$
\left.K^{2_{++} 1_{++}}\left(x, b^{\prime}\right)=\sum_{n=-\infty}^{\infty}\left\{-\sqrt{1-\alpha} \sinh \left[\frac{\left(r_{+}^{2}-r_{-}^{2}\right)}{r_{+}} \Delta t-r_{-} \Delta \phi_{n}\right)\right]+\sqrt{\alpha} \cosh \left(r_{+} \Delta \phi_{n}\right)\right\}^{-2 h_{+}} .
$$

In particular, if we consider a bulk point on the inner horizon, where $\alpha=0$, we see that the propagator remains finite; because of the $r_{-} \Delta \phi_{n}$ term in the sinh, the summand is not independent of $n$.

An $i \epsilon$ prescription for this propagator will be defined by analytic continuation from the Euclidean propagator on the metric (13). The Euclidean propagator can be obtained by making the replacements $t=-i \tau, r_{-}=-i \tilde{r}_{-}$in (16), which gives

$$
\left.K_{E}\left(x, b^{\prime}\right)=\sum_{n=-\infty}^{\infty}\left\{-\sqrt{\alpha-1} \cos \left[\frac{\left(r_{+}^{2}+\tilde{r}_{-}^{2}\right)}{r_{+}} \Delta \tau-\tilde{r}_{-} \Delta \phi_{n}\right)\right]+\sqrt{\alpha} \cosh \left(r_{+} \Delta \phi_{n}\right)\right\}^{-2 h_{+}} .
$$

One can verify by explicit calculation that this agrees with the propagator obtained by the method of images from the propagator on Euclidean $\mathrm{AdS}_{3}$; note that in the Euclidean section $\alpha=\left(r^{2}+\tilde{r}_{-}^{2}\right) /\left(r_{+}^{2}+\tilde{r}_{-}^{2}\right)$.

The bulk description of a generic field theory amplitude involves connecting a truncated Feynman diagram built from bulk-bulk propagators and vertices to the boundary points using these bulk-boundary propagators. We want to define amplitudes by analytic continuation: we therefore start with the boundary points on the the boundary of the Euclidean spacetime (13) (which is a torus), and the bulk vertices integrated over (13). We analytically continue the locations $t_{i}^{\prime}$ of the boundary points from pure imaginary back to real values, and at the same time analytically continue the parameter $r_{-}$, to obtain an amplitude in the Lorentzian field theory. We want to see how we should deform the contour of integration for the bulk vertices as we 
perform this analytic continuation. ${ }^{2}$ This will determine the $i \epsilon$ prescription for the bulk-boundary propagators (16).

We need to deform the contour to avoid singularities in the bulk-boundary propagator. The singularities in the propagator (16) are located at

$$
\Delta t=\frac{r_{+} r_{-}}{\left(r_{+}^{2}-r_{-}^{2}\right)} \Delta \phi_{n} \pm \frac{r_{+}}{\left(r_{+}^{2}-r_{-}^{2}\right)} \cosh ^{-1}\left[\sqrt{\frac{\alpha}{\alpha-1}} \cosh r_{+} \Delta \phi_{n}\right]+\frac{2 \pi r_{+}}{\left(r_{+}^{2}-r_{-}^{2}\right)} i m
$$

where $m \in \mathbb{Z}$. Note that this has the correct thermal periodicity in imaginary time.

The structure of the propagator is rather more complicated than in the nonrotating case. We would therefore first like to check that it has the correct behaviour as we take the bulk point to large distances - in this limit, we should recover the singularities of the boundary to boundary propagator discussed in [1]. In the limit $r \rightarrow \infty$, (19) reduces to

$$
\Delta t=\frac{r_{+} r_{-} \pm r_{+}^{2}}{r_{+}^{2}-r_{-}^{2}} \Delta \phi_{n}+\frac{2 \pi r_{+}}{r_{+}^{2}-r_{-}^{2}} i m
$$

If we return to the coordinate $\bar{\phi}=\phi+r_{-} t / r_{+}$which is appropriate at large distances, this becomes, after some rearrangement,

$$
\Delta t= \pm \Delta \bar{\phi}_{n}+\frac{2 \pi r_{+}}{\left(r_{+}^{2} \pm r_{+} r_{-}\right)} i m
$$

reproducing the correct lightcone singularities in the boundary to boundary propagator.

If we analytically continue $r_{-}=-i \tilde{r}_{-}$the singularities in the propagator lie at

$$
\Delta t=-i \frac{r_{+} \tilde{r}_{-}}{\left(r_{+}^{2}+\tilde{r}_{-}^{2}\right)} \Delta \phi_{n} \pm \frac{r_{+}}{\left(r_{+}^{2}+\tilde{r}_{-}^{2}\right)} \cosh ^{-1}\left[\sqrt{\frac{\alpha}{\alpha-1}} \cosh r_{+} \Delta \phi_{n}\right]+\frac{2 \pi r_{+}}{\left(r_{+}^{2}+\tilde{r}_{-}^{2}\right)} i m,
$$

where $\alpha=\left(r^{2}+\tilde{r}_{-}^{2}\right) /\left(r_{+}^{2}+\tilde{r}_{-}^{2}\right)$. If we start with the integration over the Euclidean black hole, with the external source points taken to lie at $t_{i}^{\prime}=-i \tau_{i}^{\prime}$, then the appropriate contour is from $t=0$ to $t=-i \beta$. The singularities in (22) are then of the form imaginary \pm real. That is, all the singularities associated with future (resp. past) lightlike separation are to the right (left) of our contour of integration. This will lead to a standard Feynman $i \epsilon$ prescription when we rotate back to Lorentzian signature. Note that this would not necessarily be the case if we tried to define the amplitude via an integration over imaginary $t$ at real $r_{-}$. This thus shows the importance of the analytic continuation in $r_{-}$from the point of view of this calculation.

Since we rotate $r_{-}$as well as $\tau$ in moving from the Euclidean to the Lorentzian section, it might seem that the motion of the singularities (and hence the way we need to deform our contour) is much more complicated in the present case than it was in

\footnotetext{
${ }^{2}$ The discussion of analytic continuation in the boundary field theory itself is essentially unchanged from the non-rotating case; we will therefore not repeat the discussion of propagators in the field theory contained in [1.
} 

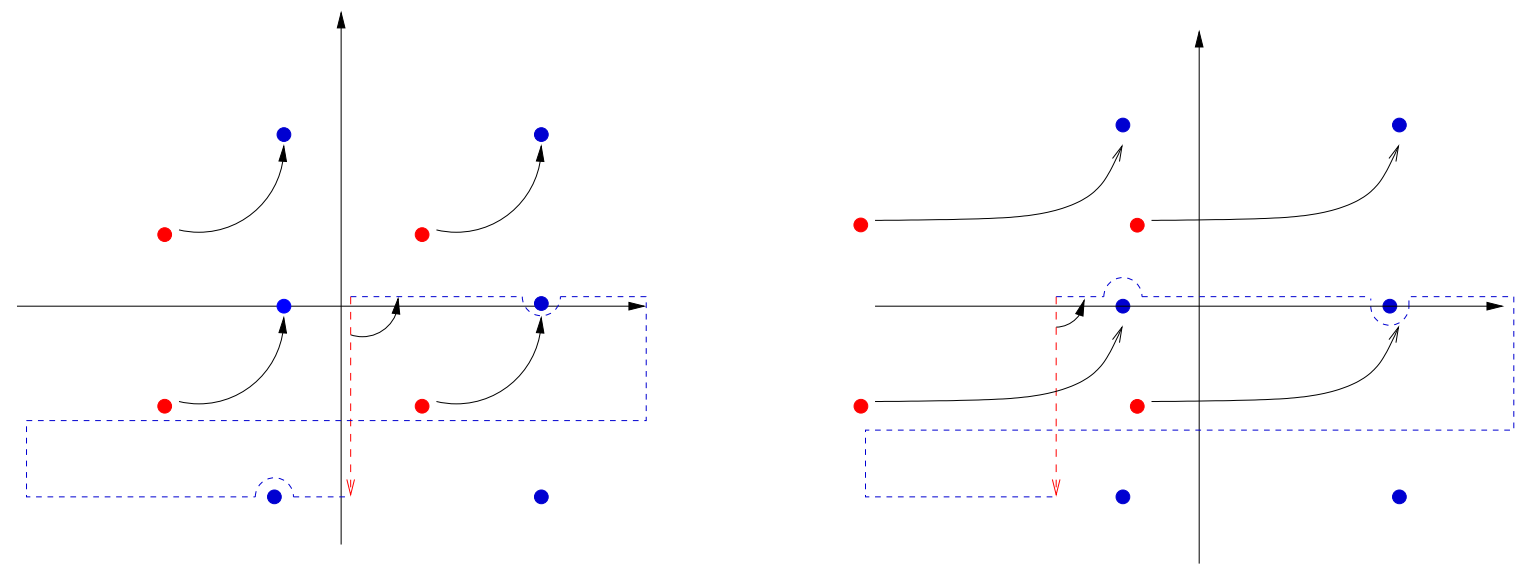

Figure 2: The analytic continuation and contour deformation in passing from the Euclidean to the Lorentzian section. The complex $z$ plane is pictured; on the right, translation invariance has been employed to move the original contour of integration to $z \in(-Z,-Z-2 \pi i)$. To simplify the figure, only singularities associated with an external point in region $1_{++}$have been indicated.

the non-rotating case. In fact, it isn't. To follow the motion of the singularities, it is convenient to define a new variable $z=\left(r_{+}^{2}-r_{-}^{2}\right) t / r_{+}$. Then the locations of the singularities in the complex $z$ plane are given by

$$
z=z^{\prime}-\tilde{r}_{-} i \Delta \phi_{n} \pm \cosh ^{-1}\left[\sqrt{\frac{\alpha}{\alpha-1}} \cosh r_{+} \Delta \phi_{n}\right]+2 \pi i m
$$

The only complication then is the behaviour of the third term. But while we want to continue from the Euclidean section, where $r_{-}=-i \tilde{r}_{-}$and $r$ is real, to the Lorentzian section, where $r_{-}$and $r$ are real, we need not keep $r$ real at intermediate stages; we can consider the analytic continuation along any path in the space of complex metrics that is convenient. Clearly, it is more convenient to perform the analytic continuation by continuing $z^{\prime}$ and $r_{-}$from imaginary to real values while keeping $\alpha$ real at intermediate stages, rather than with $r$ kept real at intermediate stages. The effect of the analytic continuation will then be to rotate the singularities onto the real axis by changing the first two terms in (23) from imaginary to real values. The rotation moves all the singularities in a counter-clockwise direction, so if we similarly move the contour of integration in a counter-clockwise direction, deforming it as illustrated in figure 2, we will obtain the usual Feynman prescription for integrating over the singularities on the light-cone.

Thus, after analytic continuation, we can deform the contour of integration for the vertices to an integration over the real $t$ axis and $t=-i \pi r_{+} /\left(r_{+}^{2}-r_{-}^{2}\right)$, and over two vertical segments connecting the horizontal segments. Since the singularities associated with external points in region $1_{++}$will appear on the real axis, while those associated with external points in region $1_{+-}$will appear on the other horizontal segment, we interpret this as saying that bulk vertices are integrated over the two regions 
$1_{+ \pm}$outside the black hole's event horizon. The vertical segments create appropriate correlations in the state on the past and future horizons on the two sides. This contour also gives us an $i \epsilon$ prescription for the bulk-boundary propagators which goes above the past singularities and below the future ones on the real axis, and vice-versa along $t=-i \pi r_{+} /\left(r_{+}^{2}-r_{-}^{2}\right)$, so it reproduces the usual thermal propagator in the region outside the black hole on each side. The addition of non-zero angular momentum therefore does not introduce any conceptual novelty relative to the discussion for the non-rotating black hole for the calculation of field theory amplitudes using this deformation of the contour.

\section{Poincare disc coordinates}

We would like to relate this calculation of the correlator by an integration over the region outside the black hole to some integral which includes the region inside the black hole. To do so, we need to find a different way of deforming the contour integral as we perform the analytic continuation. As in [1], the key is to find an appropriate coordinate system. We want an analogue of the Kruskal coordinate system used in [1], but now adapted to the identification used to construct a rotating BTZ black hole. Note that the Kruskal coordinates introduced for the rotating black hole in [19] are not apt for our purpose, as there is no explicit expression for the metric as a function of the coordinates in that coordinate system.

We can construct a coordinate system which will cover the same region of the spacetime, but in which the metric does have an explicit expression, by adopting a

different perspective on the coordinate system used in [1]. There, the metric on the Euclidean BTZ black hole was expressed as

$$
d s^{2}=\frac{4}{\left(1-\bar{X}^{2}-\bar{Y}^{2}\right)^{2}}\left[d \bar{Y}^{2}+d \bar{X}^{2}+\frac{r_{+}^{2}}{4}\left(1+\bar{X}^{2}+\bar{Y}^{2}\right)^{2} d \bar{\phi}^{2}\right],
$$

where $0 \leq \bar{X}^{2}+\bar{Y}^{2}<1$. This coordinate system is related to the embedding coordinates $\left(T_{1}, X_{1}, X_{2}, X_{3}\right)$ satisfying $T_{1}^{2}-\vec{X}^{2}=1$ by

$$
\begin{gathered}
T_{1}=\frac{1+\bar{X}^{2}+\bar{Y}^{2}}{1-\bar{X}^{2}-\bar{Y}^{2}} \cosh r_{+} \bar{\phi}, \\
X_{1}=\frac{1+\bar{X}^{2}+\bar{Y}^{2}}{1-\bar{X}^{2}-\bar{Y}^{2}} \sinh r_{+} \bar{\phi}, \\
X_{2}=\frac{2 \bar{X}}{1-\bar{X}^{2}-\bar{Y}^{2}}, \quad X_{3}=\frac{2 \bar{Y}}{1-\bar{X}^{2}-\bar{Y}^{2}} .
\end{gathered}
$$

We can recognise this coordinate system as a Cartesian version of the Poincare disc coordinates on (Euclidean) $\mathrm{AdS}_{3}$. That is, the surfaces $\bar{\phi}=$ constant in these coordinates are copies of the Poincare disc, written in a Cartesian coordinate system $\bar{X}, \bar{Y}$. If $\bar{\phi}$ is unrestricted, this is a familiar description of global $\mathrm{AdS}_{3}$. The BTZ black hole is obtained by a quotient on hyperbolic space that is expressed in these coordinates as $\bar{\phi} \sim \bar{\phi}+2 \pi$. 
Now the difference between the 'rotating' and non-rotating Euclidean black holes is simply that in the former case, we make an identification with a twist; that is, the identification which constructs the Euclidean black hole is not orthogonal to the Poincare discs in this coordinate system. A coordinate system adapted to such an identification can be created by twisting the above coordinates, i.e., through

$$
\begin{gathered}
\bar{X}=X \cos \tilde{r}_{-} \phi+Y \sin \tilde{r}_{-} \phi, \\
\bar{Y}=-X \sin \tilde{r}_{-} \phi+Y \cos \tilde{r}_{-} \phi, \\
\bar{\phi}=\phi .
\end{gathered}
$$

In these coordinates, the Euclidean metric reads

$$
\begin{aligned}
d s^{2}= & \frac{4}{\left(1-X^{2}-Y^{2}\right)^{2}}\left[d Y^{2}+d X^{2}+2 \tilde{r}_{-}(X d Y-Y d X) d \phi+\tilde{r}_{-}^{2}\left(X^{2}+Y^{2}\right) d \phi 31\right) \\
& \left.+\frac{r_{+}^{2}}{4}\left(1+X^{2}+Y^{2}\right)^{2} d \phi^{2}\right] .
\end{aligned}
$$

This coordinate system is related to the embedding coordinates by

$$
\begin{gathered}
T_{1}=\frac{1+X^{2}+Y^{2}}{1-X^{2}-Y^{2}} \cosh r_{+} \phi, \\
X_{1}=\frac{1+X^{2}+Y^{2}}{1-X^{2}-Y^{2}} \sinh r_{+} \phi, \\
X_{2}=\frac{2 X}{1-X^{2}-Y^{2}} \cos \tilde{r}_{-} \phi+\frac{2 Y}{1-X^{2}-Y^{2}} \sin \tilde{r}_{-} \phi, \\
X_{3}=-\frac{2 X}{1-X^{2}-Y^{2}} \sin \tilde{r}_{-} \phi+\frac{2 Y}{1-X^{2}-Y^{2}} \cos \tilde{r}_{-} \phi .
\end{gathered}
$$

The identification $\phi \sim \phi+2 \pi$ in these coordinates gives us the rotating BTZ black hole. Thus, this represents a convenient coordinate system on the Euclidean black hole.

Let us now analytically continue this coordinate system to Lorentzian space, setting $T=-i Y$ and $r_{-}=-i \tilde{r}_{-}$, and see what region of the black hole the resulting coordinates cover. We have a locally $\mathrm{AdS}_{3}$ Lorentzian metric

$$
\begin{aligned}
d s^{2}= & \frac{4}{\left(1-X^{2}+T^{2}\right)^{2}}\left[-d T^{2}+d X^{2}-2 r_{-}(X d T-T d X) d \phi\right. \\
& \left.-r_{-}^{2}\left(X^{2}-T^{2}\right) d \phi^{2}+\frac{r_{+}^{2}}{4}\left(1+X^{2}-T^{2}\right)^{2} d \phi^{2}\right] .
\end{aligned}
$$

This metric is now related to the Lorentzian embedding coordinates by

$$
\begin{aligned}
& T_{1}=\frac{1+X^{2}-T^{2}}{1-X^{2}+T^{2}} \cosh r_{+} \phi, \\
& X_{1}=\frac{1+X^{2}-T^{2}}{1-X^{2}+T^{2}} \sinh r_{+} \phi,
\end{aligned}
$$




$$
\begin{aligned}
& X_{2}=\frac{2 X}{1-X^{2}+T^{2}} \cosh r_{-} \phi-\frac{2 T}{1-X^{2}-Y^{2}} \sinh r_{-} \phi, \\
& T_{2}=-\frac{2 X}{1-X^{2}+T^{2}} \sinh r_{-} \phi+\frac{2 T}{1-X^{2}+T^{2}} \cosh r_{-} \phi .
\end{aligned}
$$

Thus, the relation between this coordinate system and the coordinate system of (17) is

$$
\begin{gathered}
\sqrt{\alpha}=\frac{1+X^{2}-T^{2}}{1-X^{2}+T^{2}}, \\
\tanh \left[\frac{\left(r_{+}^{2}-r_{-}^{2}\right)}{r_{+}} t\right]=\frac{T}{X}, \\
\phi=\phi .
\end{gathered}
$$

Note that this coordinate transformation is very similar to that relating the Rindler and Minkowski coordinates on flat space. This similarity is perhaps more obvious in the inverse coordinate transformation,

$$
\begin{aligned}
& X=\left(\frac{\sqrt{\alpha}-1}{\sqrt{\alpha}+1}\right)^{1 / 2} \cosh \left(\frac{\left(r_{+}^{2}-r_{-}^{2}\right)}{r_{+}} t\right), \\
& T=\left(\frac{\sqrt{\alpha}-1}{\sqrt{\alpha}+1}\right)^{1 / 2} \sinh \left(\frac{\left(r_{+}^{2}-r_{-}^{2}\right)}{r_{+}} t\right) .
\end{aligned}
$$

One would like to know what portion of the BTZ metric our rotating disc coordinates are covering. This is most easily analysed by following [1], and considering the signs of the combinations $T_{1}^{2}-X_{1}^{2}=\alpha$ and $T_{2}^{2}-X_{2}^{2}=1-\alpha$. In the coordinate system of (36), these are

$$
T_{1}^{2}-X_{1}^{2}=\left(\frac{1+X^{2}-T^{2}}{1-X^{2}+T^{2}}\right)^{2}
$$

and

$$
T_{2}^{2}-X_{2}^{2}=\frac{4\left(T^{2}-X^{2}\right)}{\left(1-X^{2}+T^{2}\right)^{2}}
$$

We see that $T_{1}^{2}-X_{1}^{2}$ is always positive, so we have only regions with $r>r_{-}$, while $T_{2}^{2}-X_{2}^{2}$ can be positive or negative, so we have regions with $r>r_{+}$or $r<r_{+}$. Exploring further, we have

$$
\begin{aligned}
T_{1} \pm X_{1} & =\frac{1+X^{2}-T^{2}}{1-X^{2}+T^{2}} e^{ \pm r_{+} \phi} \\
T_{2} \pm X_{2} & =\frac{2(T \pm X)}{1-X^{2}+T^{2}} e^{ \pm r_{-} \phi}
\end{aligned}
$$

Thus, we have the following: for $X^{2}-T^{2}>1$, we have a region $1_{-+}$and a region $1_{--}$. The surface $X^{2}-T^{2}=1$ maps to $r=\infty$. For $1>X^{2}-T^{2} \geq 0$, we have a region $1_{++}$and a region $1_{+-}$. For $0 \geq X^{2}-T^{2}>-1$, we have a region $2_{++}$and a region $2_{+-}$. This coordinate system breaks down at $X^{2}-T^{2}=-1$, as the determinant of 
the metric (36) vanishes there. For $-1>X^{2}-T^{2}>-\infty$, we have a region $2_{-+}$and a region $2_{--}$.

The physical range of coordinates is thus $1>X^{2}-T^{2}>-1$, which covers the same region as BTZ Kruskal coordinates $\left(U_{+}, V_{+}\right)$[19], namely the region including the regions $1_{+ \pm}$outside the horizon and $2_{+ \pm}$between the two horizons in one 'copy' of the BTZ black hole. Just as the regions $1_{- \pm}$are not physically connected to the regions $1_{+ \pm}$, even though they might appear to be adjacent in these coordinates, the $2_{- \pm}$ are not connected to the regions $2_{+ \pm}$in the physical spacetime (region 3 intervenes). The presence of these additional regions will be mathematically convenient in the continuation of the amplitudes, but we do not believe they should be assigned any physical significance.

Let us now consider the propagator in this coordinate system. We know from the work of [1] that the Euclidean propagator on $\mathrm{AdS}_{3}$ expressed in the coordinates (24) is

$$
K_{E}\left(x, b^{\prime}\right)=\frac{\left(1-\bar{X}^{2}-\bar{Y}^{2}\right)^{2 h_{+}}}{\left[2 \bar{X} \bar{X}^{\prime}+2 \bar{Y} \bar{Y}^{\prime}-\left(1+\bar{X}^{2}+\bar{Y}^{2}\right) \cosh ^{2} r_{+} \Delta \bar{\phi}\right]^{2 h_{+}}} .
$$

Note that since $b=\left(\bar{Y}^{\prime}, \bar{X}^{\prime}, \bar{\phi}\right)$ is meant to be a boundary point, $\bar{Y}^{\prime 2}+\bar{X}^{\prime 2}=1$. Using the coordinate transformation (28][30) to our twisted coordinates, and imposing the identification $\phi \sim \phi+2 \pi$, we find that the Euclidean black hole propagator can be re-expressed in the coordinates of (31) as

$$
\begin{aligned}
K_{E}\left(x, b^{\prime}\right)= & \sum_{n=-\infty}^{\infty}\left(1-X^{2}-Y^{2}\right)^{2 h_{+}}\left[2 X X^{\prime} \cos \tilde{r}_{-} \Delta \phi_{n}+2 Y Y^{\prime} \cos \tilde{r}_{-} \Delta \phi_{n}\right. \\
& \left.-2 X Y^{\prime} \sin \tilde{r}_{-} \Delta \phi_{n}+2 X^{\prime} Y \sin \tilde{r}_{-} \Delta \phi_{n}-\left(1+X^{2}+Y^{2}\right) \cosh r_{+} \Delta \phi_{n}\right]^{-2 h_{+}} .
\end{aligned}
$$

This can be analytically continued to give us the Lorentzian propagator in rotating disc coordinates as

$$
\begin{aligned}
K\left(x, b^{\prime}\right)= & \sum_{n=-\infty}^{\infty}\left(1-X^{2}+T^{2}\right)^{2 h_{+}}\left[2 X X^{\prime} \cosh r_{-} \Delta \phi_{n}-2 T T^{\prime} \cosh r_{-} \Delta \phi_{n}\right. \\
& \left.+2 X T^{\prime} \sinh r_{-} \Delta \phi_{n}-2 X^{\prime} T \sinh r_{-} \Delta \phi_{n}-\left(1+X^{2}-T^{2}\right) \cosh r_{+} \Delta \phi_{n}\right]^{-2 h_{+}} .
\end{aligned}
$$

Before working out the singularities in this propagator in detail, let us note an important change in the singularity structure. In the non-rotating case, there was a divergence of the summation at $X^{2}-T^{2}=-1$, which in that case corresponds to the location of the BTZ singularity. This reflected the fact that as we approach the BTZ singularity, the proper distance between different images of the bulk point in the covering space is going to zero (as is obvious from the fact that $g_{\phi \phi}$ is vanishing there). For non-zero rotation, there is no analogue of this effect, because even for $X^{2}-T^{2}=-1$, the summand does not become independent of $n$. This was already seen in BTZ coordinates in (17).

This is physically correct; in the non-rotating case, $X^{2}-T^{2}=-1$ is the BTZ singularity, and as was pointed out in [22, 20, 1, the BTZ singularity is like the singularity in the parabolic orbifold of flat space studied in [23, 25, 24, 26]. The 
divergence in the summation in the propagator near the BTZ singularity is thus related to the instability of this singularity identified in [26] (where multiple boosted images of a single particle converge). In the rotating black hole, by contrast, $g_{\phi \phi}$ does not vanish at $X^{2}-T^{2}=-1$, so the proper distance between images remains finite at $X^{2}-T^{2}=-1$; this is just a horizon in the spacetime. As discussed in the introduction, we nevertheless expect that there will be a singularity there, due to the infinite blueshift associated with this horizon, so the cutoff at the event horizon is not just an artifact of our coordinate system. The curvature singularity that forms at the Cauchy Horizon is a weak, null singularity (which is characterised by having finite tidal deformations) [27, 28]. As a result the singularity is neither associated with the sum over images or the divergence of the probe field. Hence, we should not expect it to be signalled by a failure of the summation in (52) to converge or the bulk-boundary propagator to in fact diverge at all.

Equally striking is what adding rotation does not change: there is still no imaginary periodicity in $T$. The singularities in the propagator are given by solving a quadratic in $T$, so there will be singularities at two locations in the complex plane for given $X, X^{\prime}, T^{\prime}, \Delta \phi_{n}$. Thus, perhaps surprisingly, there is no thermal behaviour associated with the inner horizon in this representation of the propagator, even though we are restricted to the region outside the inner horizon. We would argue that this is again a sign that the inner horizon is not a true regular horizon in the spacetime, because of the blueshifting instability.

Let us now determine the singularities in this Lorentzian propagator in detail. Note that the source point is by assumption located on the boundary; we established earlier that this corresponds to $X^{\prime 2}-T^{\prime 2}=1$. It is useful to introduce a new variable $\beta$ parameterising the location of the source point, writing $X^{\prime}=\eta \cosh \beta, T^{\prime}=\eta \sinh \beta$, where $\eta= \pm 1$. The singularities are then given by the roots of the quadratic in (52):

$$
\begin{array}{r}
2 X \eta \cosh \beta \cosh r_{-} \Delta \phi_{n}-2 T \eta \sinh \beta \cosh r_{-} \Delta \phi_{n}+2 X \eta \sinh \beta \sinh r_{-} \Delta \phi_{n} \\
-2 T \eta \cosh \beta \sinh r_{-} \Delta \phi_{n}-\left(1+X^{2}-T^{2}\right) \cosh r_{+} \Delta \phi_{n}=0,
\end{array}
$$

which we can simplify to

$T^{2} \cosh r_{+} \Delta \phi_{n}-2 \eta T \sinh \left(\beta-r_{-} \Delta \phi_{n}\right)-\left(1+X^{2}\right) \cosh r_{+} \Delta \phi_{n}+2 \eta X \cosh \left(\beta-r_{-} \Delta \phi_{n}\right)=0$.

The roots lie at

$$
\begin{aligned}
T= & \left(\cosh r_{+} \Delta \phi_{n}\right)^{-1}\left\{\eta \sinh \left(\beta-r_{-} \Delta \phi_{n}\right)\right. \\
& \left. \pm\left[\sinh ^{2}\left(\beta-r_{-} \Delta \phi_{n}\right)+\left(1+X^{2}\right) \cosh ^{2} r_{+} \Delta \phi_{n}-2 \eta X \cosh \left(\beta-r_{-} \Delta \phi_{n}\right) \cosh r_{+} \Delta \phi_{n}\right]^{1 / 2}\right\} .
\end{aligned}
$$

This can be further simplified to read

$$
T=\frac{\eta \sinh \left(\beta-r_{-} \Delta \phi_{n}\right) \pm\left[\left(\cosh \left(\beta-r_{-} \Delta \phi_{n}\right)-\eta X \cosh r_{+} \Delta \phi_{n}\right)^{2}+\sinh ^{2} r_{+} \Delta \phi_{n}\right]^{1 / 2}}{\cosh r_{+} \Delta \phi_{n}}
$$

Note that the two singularities in the complex $T$ plane lie on the real axis, as they should; these are just light-cone singularities. 
We would now like to study the contour deformation for the vertex using this coordinate system, and thereby obtain an $i \epsilon$ prescription for the Lorentzian propagator (52). As in [1], there is a technical difficulty in that the natural range of integration in the Euclidean metric is $0 \leq X^{2}+Y^{2} \leq 1$, and we do not understand how to perform an analytic continuation subject to this kind of restriction. However, the propagator (51) is symmetric under the antipodal map

$$
X \rightarrow \frac{X}{X^{2}+Y^{2}}, \quad Y \rightarrow \frac{Y}{X^{2}+Y^{2}}
$$

so long as $2 h_{+}$is even. Hence, at least in this case, we can treat the region $X^{2}+Y^{2}>1$ as another copy of the black hole solution, and obtain the correct answer for the amplitude by integrating over the full $X, Y$ plane and then dividing the final answer by two.

Let us therefore consider an integration over the Euclidean section, where the source points lie on the circle $X^{\prime 2}+Y^{\prime 2}=1$, and the integration over $T$ runs from $T=i \infty$ to $T=-i \infty$. The singularities then lie initially at

$T=\frac{-i \eta \sin \left(\tilde{\beta}-\tilde{r}_{-} \Delta \phi_{n}\right) \pm\left[\left(\cos \left(\tilde{\beta}-\tilde{r}_{-} \Delta \phi_{n}\right)-\eta X \cosh r_{+} \Delta \phi_{n}\right)^{2}+\sinh ^{2} r_{+} \Delta \phi_{n}\right]^{1 / 2}}{\cosh r_{+} \Delta \phi_{n}}$,

where $\tilde{\beta}$ is defined by $X^{\prime}=\eta \cos \tilde{\beta}, Y^{\prime}=\eta \sin \tilde{\beta}$. As in the BTZ coordinates, we see that the singularities associated with the Euclidean solution lie at imaginary \pm real, so that the singularities associated with future (resp. past) lightlike separation are to the right (left) of our contour of integration.

The analytic continuation here looks still more complicated than in the previous case; however, once again we can avoid complications in the motion of the singularities by a convenient choice of path. Instead of keeping $X$ real at intermediate stages in the analytic continuation, it is better to assume we keep $\cosh \left(\beta-r_{-} \Delta \phi_{n}\right)-\eta X \cosh r_{+} \Delta \phi_{n}$ real. The analytic continuation then involves only the first term in (156), and will rotate the singularities counter-clockwise. If we similarly rotate the contour counterclockwise, we will wind up with the usual $i \epsilon$ prescription, where we make the substitution $Y=e^{i(\pi / 2-\epsilon)} T$ in (51). The analytic continuation is thus accurately represented by the simple picture given in figure 3 .

We are left with an integration over the full $X, T$ plane for each vertex. However, as we saw above, the full $X, T$ plane contains regions $1_{- \pm}$and $2_{- \pm}$, as well as the regions $1_{+ \pm}, 2_{+ \pm}$of interest. It is thus a double cover of a single Kruskal patch $r \geq r_{-}$ in the Lorentzian black hole. We can simply cancel this double cover against the factor of a half that came from the double cover we introduced in the Euclidean black hole, and obtain an integration just over the region $-1 \leq X^{2}-T^{2}<1$ corresponding to a single copy of the Kruskal patch. As in [1], this will lead to a slightly more complicated $i \epsilon$ prescription in the regions $r_{-} \leq r \leq r_{+}$.

The main point is that, by analytic continuation, we can express the bulk spacetime version of the calculation of a CFT amplitude either in terms of an integration of the interaction vertices over regions $1_{++}$and $1_{+_{-}}$in the rotating black hole, with the $i \epsilon$ prescription given in the previous section, or in terms of an integration over regions 


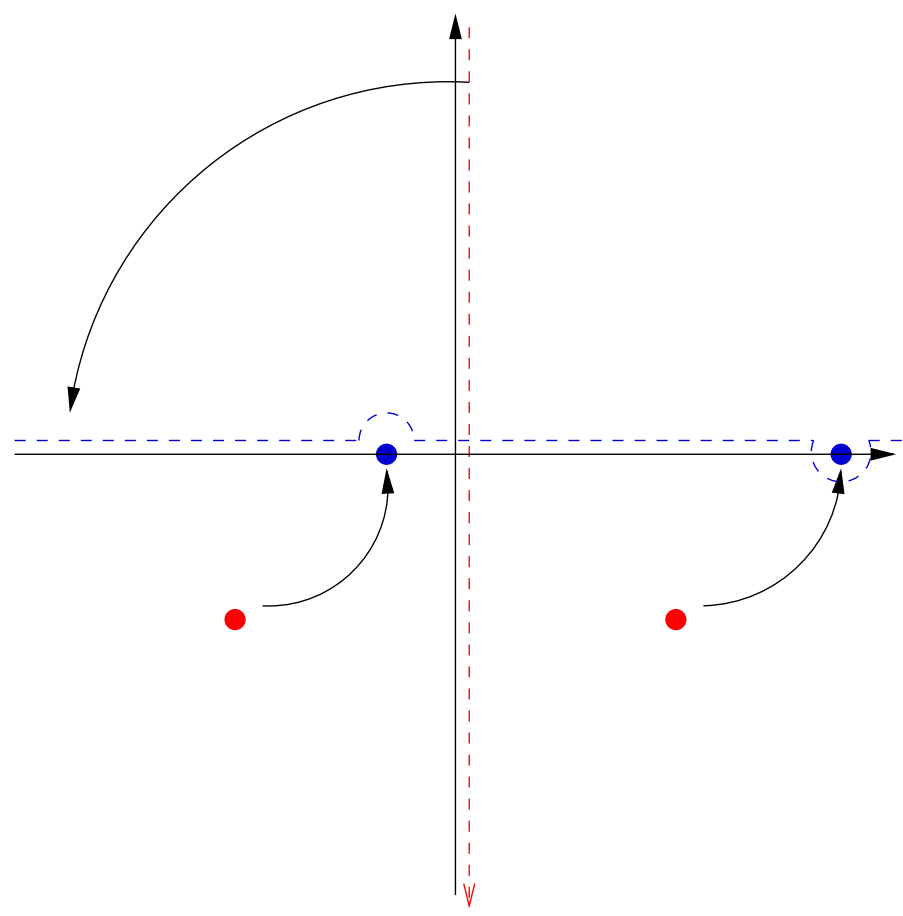

Figure 3: The analytic continuation and contour deformation in passing from the Euclidean to the Lorentzian section, in Poincare disc coordinates.

$1_{+ \pm}$and $2_{+ \pm}$, with the $i \epsilon$ prescription given here. Thus, such amplitudes involve the region behind the event horizon, but outside of the Cauchy horizon.

\section{Conclusions}

Our main objective in this paper has been to deepen our understanding of the relation between the field theory and the spacetime in the AdS/CFT correspondence for spacetimes with horizons. We have shown that in rotating BTZ, the bulk calculation of field theory amplitudes defined by analytic continuation can be expressed in two ways, in terms of an integral over the region outside the horizon, or in terms of an integral over the region bounded by past and future Cauchy horizons. This extends previous work [1] to include rotation.

A feature which deserves to be stressed again is that the analytic continuation from the Euclidean black hole gives us an integral over both asymptotic regions, $1_{++}$ and $1_{+-}$. In particular, the analytic continuation of the single boundary in Euclidean BTZ gives us the two boundaries in Lorentzian BTZ. In the original coordinate system, this arises because the deformed contour defining the real-time thermal field theory involves two horizontal segments, interpreted as independent fields on the two boundaries that are entangled by the state of the CFT. In the Poincare disc coordinates, we pass from the connected boundary $X^{2}+Y^{2}=1$ in Euclidean space to the two-sheeted boundary $X^{2}-T^{2}=1$ in Lorentzian space. The black hole is thus still 
represented by a pure state - an entangled state of the two field theories. It seems natural that such a pure state will contain complete information about the bulk spacetime. If we trace over the degrees of freedom on one boundary, the resulting thermal state on the other boundary will no longer contain enough information to describe the region behind the horizon.

The main value of adding rotation is that it highlights the importance of the Cauchy horizon as a boundary. We have seen that amplitudes can be related to an integral over the region bounded by the Cauchy horizons. For Lorentzian AdS, a state in the field theory is conjectured [14] to be dual to the initial state in the bulk AdS spacetime, encoded by data on some Cauchy surface. It therefore seems very natural that the calculation of amplitudes in the field theory involves the region determined by this initial data. We conjecture that in general, the CFT description is not limited to the spacetime region which is visible from infinity - that is, it does not stop at event horizons. It stops only when we encounter a Cauchy horizon. ${ }^{3}$

This conjecture gains further support from carefully considering the horizon in the Poincare coordinates on pure AdS. In [29], it was found that the region behind the horizon in Poincare coordinates is included in the CFT description, because the CFT naturally lives on the whole Einstein static universe $\mathbb{R} \times S^{n}$, and not just in Minkowski space $\mathbb{R}^{n, 1}$. Put another way, the CFT on $\mathbb{R}^{n, 1}$ determines the bulk spacetime within the Poincare horizon; to go beyond it, we need to fix boundary conditions on other parts of the boundary of AdS. The boundary of the Poincare patch is an (observerdependent) event horizon, but it is also the Cauchy horizon for a $t=$ constant surface in Poincare coordinates (supplemented by the boundary conditions supplied by the field theory on $\mathbb{R}^{n, 1}$ ). Thus, the result of [29], that an extension of the field theory (from $\mathbb{R}^{n, 1}$ to $\mathbb{R} \times S^{n}$ ) is necessary to describe the region of spacetime beyond the Poincare horizon, is perfectly consonant with relating the field theory to the region bounded by the Cauchy horizons.

In fact, our consideration of the rotating BTZ black hole is very close in spirit to the analysis of [29]. The periodic identification in $\phi$ that defines the black hole has played only a spectator role in our discussion. Thus, one could equally well apply our analysis to AdS in BTZ coordinates without the identification along $\phi$. We would then say that what we have learnt is that if one considers the the field theory just on those regions of the boundary where $\partial_{\phi}$ is spacelike, it is dually related to the region of the spacetime which is determined by Cauchy data supplemented by the asymptotic boundary conditions on this portion of the boundary. So the discussion here is in a sense a generalisation of 29], considering the restriction to a different region of the boundary.

If the field theory only describes the region of spacetime before the Cauchy horizon, what is the physical interpretation of the region beyond that horizon? In some special cases, such as the Poincare coordinates in pure AdS, this is an unnatural restriction, and the spacetime beyond the horizon can be included in the description by an extension of the field theory which supplies the necessary boundary conditions. However,

\footnotetext{
${ }^{3} \mathrm{~A}$ similar argument that the Lorentzian correspondence requires that some regions beyond event horizons be included in the CFT description was made in [10].
} 
we will further conjecture that generically there will be no such extension - the region beyond the Cauchy horizon is not included in the 'hologram', so it is unphysical. Thus, the AdS/CFT correspondence enforces strong cosmic censorship for such solutions [16. The classical description will break down at the Cauchy horizon, so no observer can study quantum effects from a safe distance.

The idea that the spacetime is cut off at the Cauchy horizon receives support from previous calculations, which have shown that an observer approaching the Cauchy horizon inside a rotating black hole will generically encounter a divergent flux of radiation due to the infinite blueshift [18]. Thus, the classical theory does predict its own downfall there. This should be contrasted with the situation at the event horizon, where an observer crossing the horizon will see nothing special. An important direction for future work is to understand to what extent this blueshifting instability is reflected in the CFT calculations we have discussed here, and more generally to see evidence for the conjectured breakdown of the classical spacetime description at the Cauchy horizon and understand what replaces it.

If the event horizon is not a fundamental barrier, why does it remain so difficult to answer questions about physics there? One part of the answer to this question, as pointed out in [1, is that the size of a black hole, from the viewpoint of an infalling observer, is never greater than the characteristic curvature scale of the AdS space. This remains true for rotating black holes: the proper time taken to fall from the outer to the inner event horizon of a rotating black hole is bounded from above by a number of order one in $\mathrm{AdS}$ units for any value of $r_{ \pm}$. Hence, one obstacle to understanding the observations of infalling observers is that we do not know how to recover approximately local bulk physics on scales smaller than the AdS scale from the field theory. Making progress on this issue will be essential to gain a better understanding of the description of the black hole's geometry in the field theory.

Another barrier to understanding is that the part of the connection between bulk and boundary we understand well is the relation of the asymptotic behaviour of fields in spacetime and the expectation values of local operators in the field theory. This can be used indirectly to understand some features of the description of sources in the bulk in terms of their effects at large distances, but does not tell us how the CFT describes changes in the bulk whose effects have not yet propagated to the boundary. Understanding the CFT degrees of freedom which describe the bulk more directly is the problem of precursors [30, 31, 32, 33. Precursors are supposed to reflect events deep inside the spacetime without (immediately) changing any local observables in the CFT. The region behind the black hole's event horizon provides a particularly sharp example of this problem, as events in this region can never effect the asymptotic region, so they will only ever be reflected in the CFT through non-local observables tied to the precursors. This point was also discussed in [10]. Thus, understanding the description of changes behind the event horizon will also require progress on our understanding of the direct description of the interior of spacetime in the dual CFT. The present calculation reinforces the arguments that the region inside the event horizon is included in the CFT description, but it does not address these deep questions concerning how that description is implemented.

Finally, it would be interesting to explore the extension of these calculations to 
higher dimensions. This will clearly be more challenging, as the construction of suitable coordinate systems in our work here has relied on cunningly exploiting the locally AdS character of the BTZ black holes.

\section{Acknowledgements}

We thank Vijay Balasubramanian and Asad Naqvi for extensive discussions. T.S.L. is supported by the DOE under cooperative research agreement DE-FG02-95ER40893. S.F.R. is supported by an EPSRC Advanced Fellowship.

\section{References}

[1] P. Kraus, H. Ooguri and S. Shenker, "Inside the horizon with AdS/CFT," arXiv:hep-th/0212277.

[2] J. M. Maldacena, "The large $N$ limit of superconformal field theories and supergravity," Adv. Theor. Math. Phys. 2 (1998) 231 [Int. J. Theor. Phys. 38 (1999) 1113] arXiv:hep-th/9711200.

[3] E. Witten, "Anti-de Sitter space, thermal phase transition, and confinement in gauge theories," Adv. Theor. Math. Phys. 2 (1998) 505 arXiv:hep-th/9803131.

[4] G. T. Horowitz and S. F. Ross, "Possible resolution of black hole singularities from large $N$ gauge theory," JHEP 9804 (1998) 015 arXiv:hep-th/9803085.

[5] L. Susskind and L. Thorlacius, "Gedanken experiments involving black holes," Phys. Rev. D 49 (1994) 966 arXiv:hep-th/9308100.

[6] L. Susskind, L. Thorlacius and J. Uglum, "The Stretched horizon and black hole complementarity," Phys. Rev. D 48, 3743 (1993) arXiv:hep-th/9306069.

[7] V. Balasubramanian and S. F. Ross, "Holographic particle detection," Phys. Rev. D 61 (2000) 044007 arXiv:hep-th/9906226.

[8] J. Louko, D. Marolf and S. F. Ross, "On geodesic propagators and black hole holography," Phys. Rev. D 62 (2000) 044041 arXiv:hep-th/0002111.

[9] J. M. Maldacena, "Eternal black holes in Anti-de-Sitter," arXiv:hep-th/0106112.

[10] V. E. Hubeny, "Precursors see inside black holes," arXiv:hep-th/0208047.

[11] W. Israel, "Thermo Field Dynamics Of Black Holes," Phys. Lett. A 57 (1976) 107.

[12] J. M. Maldacena and A. Strominger, "AdS 3 black holes and a stringy exclusion principle," JHEP 9812 (1998) 005 arXiv:hep-th/9804085.

[13] G. T. Horowitz and D. Marolf, "A new approach to string cosmology," JHEP 9807 (1998) 014 arXiv:hep-th/9805207. 
[14] V. Balasubramanian, P. Kraus and A. E. Lawrence, "Bulk vs. boundary dynamics in anti-de Sitter spacetime," Phys. Rev. D 59 (1999) 046003 arXiv:hep-th/9805171. V. Balasubramanian, P. Kraus, A. E. Lawrence and S. P. Trivedi, "Holographic probes of anti-de Sitter space-times," Phys. Rev. D 59 (1999) 104021 arXiv:hep-th/9808017.

[15] M. Berkooz, B. Craps, D. Kutasov and G. Rajesh, "Comments on cosmological singularities in string theory," JHEP 0303 (2003) 031 arXiv:hep-th/0212215.

[16] R. Penrose, "Gravitational Collapse: The Role Of General Relativity," Riv. Nuovo Cim. 1 (1969) 252 [Gen. Rel. Grav. 34 (2002) 1141]; R. Penrose, "Singularities and time-asymmetry," in General Relativity, an Einstein Centenary Survey, eds. S.W. Hawking and W. Israel (Cambridge University Press, 1979).

[17] R. Penrose, in Batelle Rencontres, eds. C. de Witt and J. Wheeler (W.A. Benjamin, New York, 1968), p. 222.

[18] R. A. Matzner, N. Zamorano and V. D. Sanberg, "Instability of the Cauchy horizon of Reissner-Nordström black holes," Phys. Rev. D 19, 2821 (1979); S. Chandrasekhar and J. Hartle, "On crossing the Cauchy horizon of a ReissnerNordström black hole," Proc. Roy. Soc. Lond. A384, 301 (1982).

[19] M. Banados, M. Henneaux, C. Teitelboim and J. Zanelli, "Geometry of the $(2+1)$ black hole," Phys. Rev. D 48, 1506 (1993) arXiv:gr-qc/9302012.

[20] S. Hemming, E. Keski-Vakkuri and P. Kraus, "Strings in the extended BTZ spacetime," JHEP 0210 (2002) 006 arXiv:hep-th/0208003.

[21] E. Keski-Vakkuri, "Bulk and boundary dynamics in BTZ black holes," Phys. Rev. D 59, 104001 (1999) arXiv:hep-th/9808037.

[22] E. J. Martinec and W. McElgin, "Exciting AdS orbifolds," JHEP 0210 (2002) 050 arXiv:hep-th/0206175.

[23] H. Liu, G. Moore and N. Seiberg, "Strings in a time-dependent orbifold," JHEP 0206 (2002) 045 arXiv:hep-th/0204168; H. Liu, G. Moore and N. Seiberg, "Strings in time-dependent orbifolds," JHEP 0210 (2002) 031 arXiv:hep-th/0206182.

[24] A. Lawrence, "On the instability of 3D null singularities," JHEP 0211 (2002) 019 arXiv:hep-th/0205288.

[25] M. Fabinger and J. McGreevy, "On smooth time-dependent orbifolds and null singularities," arXiv:hep-th/0206196.

[26] G. T. Horowitz and J. Polchinski, "Instability of spacelike and null orbifold singularities," Phys. Rev. D 66 (2002) 103512 [arXiv:hep-th/0206228. 
[27] E. Poisson and W. Israel, "Internal Structure Of Black Holes," Phys. Rev. D 41 (1990) 1796.

[28] A. Ori, "Inner structure of a charged black hole: An exact mass-inflation solution" Phys. Rev. Lett. 67 (1991) 789.

[29] G. T. Horowitz and H. Ooguri, "Spectrum of large N gauge theory from supergravity," Phys. Rev. Lett. 80 (1998) 4116 arXiv:hep-th/9802116.

[30] J. Polchinski, L. Susskind and N. Toumbas, "Negative energy, superluminosity and holography," Phys. Rev. D 60 (1999) 084006 arXiv:hep-th/9903228.

[31] L. Susskind and N. Toumbas, "Wilson loops as precursors," Phys. Rev. D 61 (2000) 044001 arXiv:hep-th/9909013.

[32] S. B. Giddings and M. Lippert, "Precursors, black holes, and a locality bound," Phys. Rev. D 65 (2002) 024006 arXiv:hep-th/0103231|.

[33] B. Freivogel, S. B. Giddings and M. Lippert, "Toward a theory of precursors," Phys. Rev. D 66 (2002) 106002 arXiv:hep-th/0207083]. 